\title{
Surgery for acute type A aortic dissection
}

\author{
Tirone E. David, MD
}

See related article on pages 294-301.

Acute type A dissection is often lethal unless emergency surgery is done. I suspect that most patients with acute type A aortic dissections are operated on by surgeons with limited experience in aortic surgery, which may explain why the operative mortality and morbidity remain high for this disease. ${ }^{1-4}$ Referring these patients to experienced surgeons may be inappropriate, because whatever might be gained in reducing the operative risk could be lost in preoperative mortality by delaying surgery. In addition, even experienced aortic surgeons are humbled by type A aortic dissection, because their results are not always stellar either. This is an operation that every cardiac surgeon should be able to perform, and if it is planned and executed well, the mortality and morbidity rates can be reduced. ${ }^{5,6}$

This review on technical aspects of surgery for acute type A aortic dissection was written with the "general cardiac surgeon" in mind, the one who sees only a few patients with this problem each year. On the basis of my experience in teaching surgical trainees, I will be writing what I believe such a general aortic surgeon should do, rather than what I do.

Prompt diagnosis and timing of surgery are crucial in this disease, and the sooner you can get the patient on the operating table, the better the operative and long-term outcomes will be.

\section{THE HEMODYNAMICALLY UNSTABLE PATIENT}

The patient in hemodynamically unstable condition probably has pericardial tamponade or acute myocardial ischemia as a result of the dissection and often is receiving inotropes and vasopressors. An arterial line on the right radial or brachial artery, a central venous line, and a good peripheral intravenous access are indispensable. You should start the operation by exposing the anterior wall of the femoral vessels. If you are familiar with the Seldinger technique, put a purse-string suture on the anterior wall of each of these vessels. If you are unfamiliar with this technique, or

\footnotetext{
From the Division of Cardiovascular Surgery of the Peter Munk Cardiac Centre at Toronto General Hospital and University of Toronto, Toronto, Ontario, Canada. Disclosures: Author has nothing to disclose with regard to commercial support. Received for publication May 29, 2015; accepted for publication June 3, 2015. Address for reprints: Tirone E. David, MD, 200 Elizabeth St 4N453, Toronto, ON M5G 2C4 Canada (E-mail: tirone.david@uhn.ca).

J Thorac Cardiovasc Surg 2015;150:279-83

$0022-5223 / \$ 36.00$

Copyright (c) 2015 by The American Association for Thoracic Surgery http://dx.doi.org/10.1016/j.jtcvs.2015.06.009
}

if you don't have the appropriate perfusion cannulas, or if the femoral artery has evidence of dissection, mobilize the vessels circumferentially and put tapes around then. Next, go to the chest and do a full median sternotomy. If the hemodynamic instability is due to tamponade, the pericardial cavity will be tense and blue. Don't open it, because if you do there is a risk of a hypertensive crisis, and the aorta may blow up and make things messy. Give heparin and insert perfusion cannulas in the femoral vessels. Both the arterial and venous cardiopulmonary bypass lines should have a second arm of tubing 80 to $100 \mathrm{~cm}$ long, in case you need another venous cannula to completely drain the right side of the heart, or another arterial cannula. Start bypass and start to cool the patient. Make a small incision in the pericardium and begin to drain the fluid slowly. Alert the anesthetist about the possibility of hypertension and reduce the doses of inotropes and vasopressors. The rupture that caused the tamponade is often sealed, and there is no active bleeding in most patients. If this is the case, carry on, and you may want to insert a cannula in the right atrium for better venous drainage. The heart is not likely to fibrillate very soon if left undisturbed, because it is the last organ to receive cold blood when blood is pumped into a femoral artery. If the heart is ischemic because of occlusion of the right coronary artery by the dissection or if there is severe aortic insufficiency, however, the heart may fibrillate sooner than you would hope. Regardless, it is safer to insert a vent into the right superior pulmonary vein and gently advance it into the left ventricle. In addition, you should do 2 more things while the patient is being cooled: insert a cardioplegia cannula into the coronary sinus and put a tape around the superior vena cava. If the heart begins to fibrillate but the left ventricle is not distending, wait until the nasopharyngeal temperature reaches $20^{\circ} \mathrm{C}$ to $22^{\circ} \mathrm{C}$. If the ventricle is distending, apply suction on the ventricular vent and increase the pump flow. Uncommonly, the aortic insufficiency is so severe that perfusion pressure cannot be maintained or the heart remains distended and requires more than an occasional manual squeeze to decompress it. In that case, reduce the pump flow to $1 \mathrm{~L} / \mathrm{min}$ and apply a large clamp on the proximal half of the ascending aorta, as close to the sinotubular junction as possible without much dissection (you will have to clamp part of the pulmonary artery too), and gently increase the pump flow. When the heart is empty, give 1 to 2 $\mathrm{L}$ of cold blood cardioplegia into the coronary sinus. 
When the target temperature is reached, snare the superior vena cava and wait for 30 seconds before stopping the pump (venous hypertension prevents air from entering the cerebral circulation). Transect the ascending aorta at its middle portion and incise its anterior wall (false and true lumens) up to the level of the innominate artery. Visually inspect the intima of the aortic arch for any tear, and if there is none, cut the ascending aorta a few millimeters below the level of the innominate artery.

If you did not have to clamp the aorta because of severe aortic insufficiency, now is the time to give retrograde blood cardioplegia (the perfusionist has to know in advance that cardioplegia will be given during circulatory arrest).

Choose a tubular Dacron polyester fabric graft that fits inside the true lumen of the arch, usually 26 to $30 \mathrm{~mm}$ in diameter, depending on the sizes of the patient and aorta. Next, suture the graft 5 to $7 \mathrm{~mm}$ inside the intima on the posterior wall of the aortic arch with a horizontal mattress suture of 4-0 polypropylene buttressed on a 4- to 5-mm wide strip of polytetrafluoroethylene felt on the adventitia. The needle should be the thinnest you can find. Begin to suture the graft into the posterior wall of the arch by making sure that the graft lies at least $5 \mathrm{~mm}$ inside the dissected intima of the aortic arch. Every bite should be through the intima first, 3 to $4 \mathrm{~mm}$ apart from each other and 5 to $7 \mathrm{~mm}$ into the arch and in the graft. Once you reach the innominate artery, take the second arm of the suture and begin to sew the anterior wall of the graft inside the intima with the same precision that you used to suture the posterior wall. Never pull the suture against the intima; always pull it against the graft to prevent tears in the intima along the suture line. I do not think that you should use French glue or BioGlue (CryoLife, Inc, Kennesaw, Ga), but other experienced surgeons believe that this makes the anastomosis more hemostatic. If you are going to use glue, do so sparingly and only in between the dissected layers along the first centimeter where you are going to suture the graft. Fibrin glue can also be used to "glue" the layers together before suturing the graft, and it does not cause tissue necrosis the way that the others do. Next, remove the arterial femoral cannula and insert it into the Dacron polyester fabric graft $1 \mathrm{~cm}$ from the distal anastomosis by making a small transverse cut in the graft (smaller than the size of the arterial cannula) and placing 2 4-0 polypropylene sutures, 1 on each end of the cut to secure the cannula and later to close the hole in the graft. Start antegrade perfusion with an open graft and carefully deair the aortic arch. Clamp the graft close to the new arterial cannula, and increase the perfusion pressure. Release the tape on the superior vena cava, and reestablish full cardiopulmonary bypass. The distal anastomosis seldom leaks if it is performed as described. If it does leak, place extra stitches but reduce the pump flow to $1 \mathrm{~L} / \mathrm{min}$ while you are putting in the stitches and tying them. When the anastomosis is perfect, begin to rewarm the patient. Figure 1 illustrates the sequence of the technique described here.

I have had a few patients in whom the ascending aorta was bleeding actively when I opened the pericardium, and in these cases I had to exsanguinate the patient into the venous reservoir, clamp the aorta above the rupture, and reestablish full cardiopulmonary bypass before proceeding with the operation as described previously. Those patients did not always survive surgery.

Clamping the aorta just below the takeoff of the innominate artery in acute type A aortic dissection should be avoided when arterial blood is pumped in the femoral artery, because it can increase the risk of malperfusion and cause multiple ruptures of the false lumen (Figure 1).

It is generally believed that it is important to resect the primary tear in type A aortic dissection. Luckily, in most patients the tear starts just above the sinotubular junction in the greater curvature of the ascending aorta. The primary tear may be in the aortic arch, however, and resection can be complicated because it may require replacement of the entire aortic arch, and sometimes even replacement of the proximal parts of the brachiocephalic arteries. This is not an operation for a "general cardiac surgeon." I believe that the safest thing for you to do in this case is to replace the ascending aorta as described previously and leave the tear in the aortic arch, unless you can resect the tear without detaching the brachiocephalic vessels from the aortic arch and descending thoracic aorta. You should do your best to save the patient's life, and if further surgery is needed in the future, so be it; it is certainly much better than death or a devastating stroke if you try to replace the aortic arch and brachiocephalic arteries in this acute setting.

\section{THE HEMODYNAMICALLY STABLE PATIENT}

When the patient is in hemodynamically stable condition, you can take a bit more time and do things differently. You should have 2 monitoring arterial lines, 1 on each arm, and perhaps even a third line in a femoral artery (all this to control malperfusion during cardiopulmonary bypass). If you know how to expose the right axillary artery, this is the ideal vessel to use as the arterial return during cardiopulmonary bypass, unless there is a complex arch dissection with tears that extend into the innominate artery, which is uncommon. Aortic dissection seldom extends into the right axillary artery, but when it does you should not use this artery for the arterial return. The artery should be mobilized for a length of 25 to $30 \mathrm{~mm}$ and have elastic vessel loops passed around it proximally and distally, or you also can use fine vascular clamps. A tubular graft of 6- or 8-mm (Dacron polyester fabric or Gore-Tex [W. L. Gore \& Associates, Inc, Newark, Del]) should be sutured to it in an end-toside fashion and used for arterial return (Figure 2). You do not have to give heparin before performing this anastomosis, because there are plenty of collaterals around the 

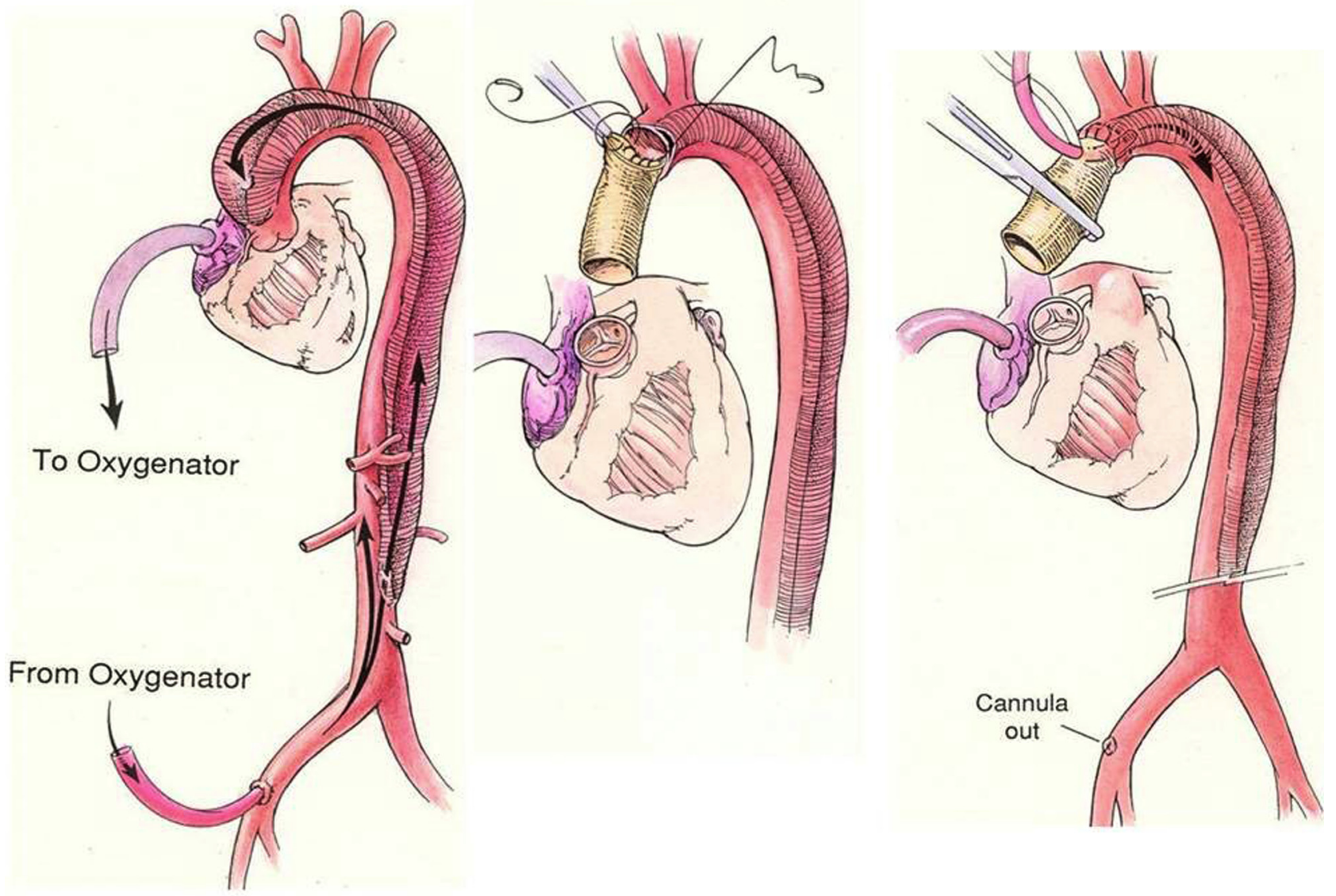

FIGURE 1. Surgery for acute type A aortic dissection. Femoral artery perfusion reverses the flow in the false lumen, and aortic clamping should be avoided to reduce the risk of malperfusion. The distal anastomosis should be performed under circulatory arrest. The femoral cannula should be removed and inserted into the Dacron polyester fabric graft for antegrade perfusion after completion of the distal anastomosis.

axillary artery. Once the anastomosis has been completed, release the distal vessel loop first and let some blood out. Occlude the vessel distally and release the proximal vessel loop for a fraction of second. Clamp the graft a few millimeters from the anastomosis and release both vessel loops. Make sure that the anastomosis is intact. The blood pressure in the right radial artery should return to normal after the release of the vessel loops.

Do a full median sternotomy, open the pericardium, and give heparin. Connect the arterial line to the axillary artery graft (Figure 2). This line should have a second limb of tubing 80 to $100 \mathrm{~cm}$ long for another arterial cannula if needed. Use the right atrium for venous drainage. Initiate cardiopulmonary bypass and began to cool the patient. If the mean arterial pressure in the right arm is greater than that in the left arm by more than 15 to $20 \mathrm{~mm} \mathrm{Hg}$, gently snare the distal vessel loop on the axillary artery until the mean pressures are similar. If the arterial resistance in the pump head is too high to deliver the calculated blood flow, you need to insert a second arterial cannula, probably in the femoral artery. Insert a left ventricular vent through the right superior pulmonary vein. You can now clamp the ascending aorta 1 to $2 \mathrm{~cm}$ below the takeoff of the innominate artery and wait for a few seconds to make sure that the blood pressure remains the same in both arms. Transect the aorta and give cardioplegia either directly into the coronary arteries or retrograde into the coronary sinus.

Carefully dissect the aortic arch and brachiocephalic arteries. Start by detaching the pericardial reflection around the distal ascending aorta, then the innominate vein, and finally the 3 brachiocephalic arteries. When the nasopharyngeal temperature is $25^{\circ} \mathrm{C}$ and the rectal temperature is below $30^{\circ} \mathrm{C}$ (the rectal temperature will not drop as fast as the nasopharyngeal temperature because cold blood is being pumped into the axillary artery), you can reduce the flow to $10 \mathrm{~mL} / \mathrm{kg} / \mathrm{min}$, clamp all 3 brachiocephalic vessels, remove the aortic clamp, and cut the ascending aorta (true and false lumens) immediately below the level of the innominate artery. Suture a tubular Dacron polyester fabric graft to the aortic arch as described previously for the patient in hemodynamically unstable condition. Once this anastomosis has been completed, you may use the axillary artery for antegrade perfusion or insert a new arterial cannula into the Dacron polyester fabric graft as described 

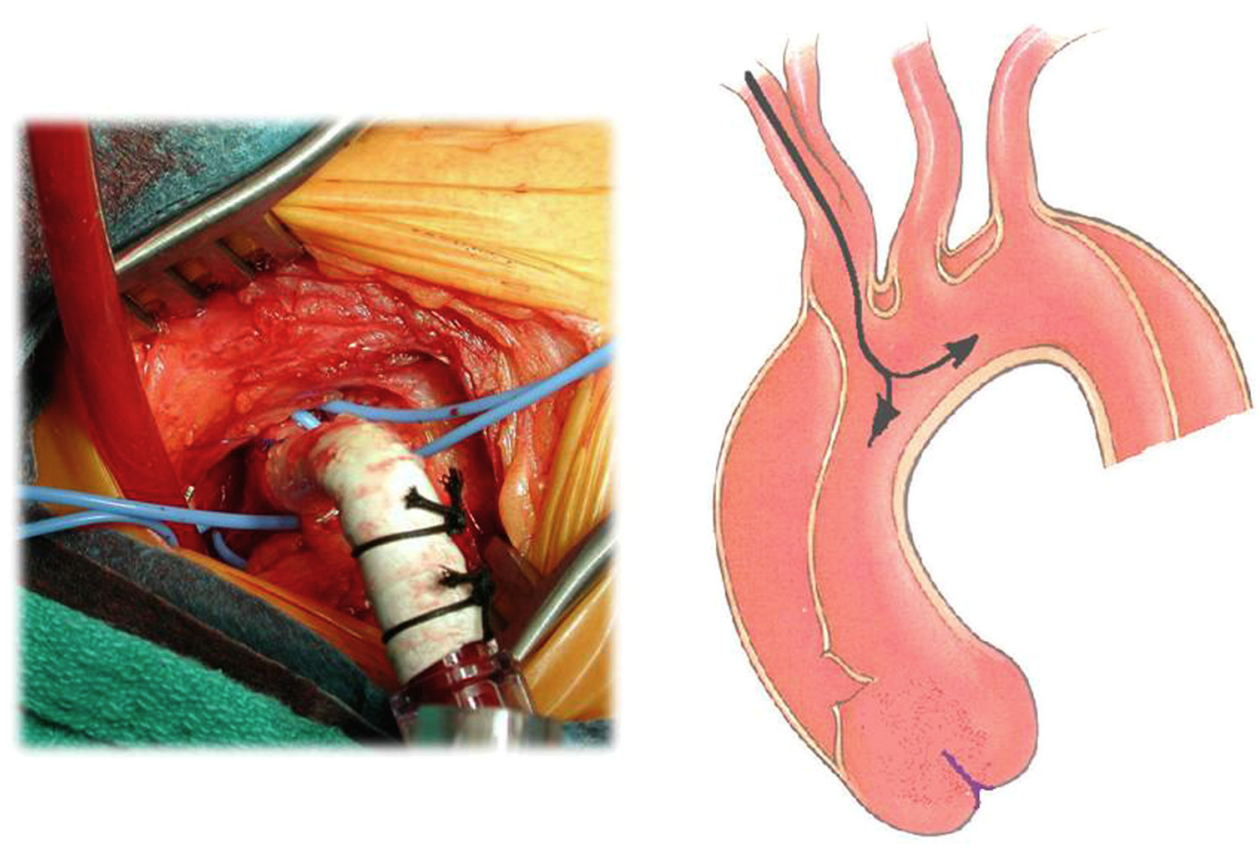

FIGURE 2. Axillary artery perfusion may prevent malperfusion because blood is pumped into the true lumen. With this type of arterial return during cardiopulmonary bypass, the ascending aorta may be clamped without increasing the risk of malperfusion.

previously. If the anastomosis is intact, begin to rewarm the patient.

If you are unfamiliar with axillary artery anatomy, or this artery is too small to supply blood to the entire body, you may use the femoral artery for cardiopulmonary bypass and conduct the operation as described for the patient in hemodynamically unstable condition, but avoid clamping the aorta to reduce the risks of malperfusion and further expansion of the false lumen.

\section{THE AORTIC ROOT}

Once the distal anastomosis has been completed, attention is turned to the aortic root. Transect the false and true lumens 5 to $7 \mathrm{~mm}$ above the sinotubular junction. Gently suspend the 3 commissures of the aortic valve and inspect the cusps. If they are normal or nearly normal, an attempt should be made to save them. Next, inspect the aortic sinuses and the extensiveness of the dissection. If it involves only the noncoronary aortic sinus and part of the right (the most common finding in acute type A dissection) and the sinuses are not aneurysmal, the sinuses can be preserved. I don't believe that you should use glue (except for fibrin glue); however, others disagree with me. If you are going to use glue, do so sparingly to minimize tissue necrosis and false aneurysms later on. Suspend the 3 commissures and approximate them until the 3 cusps touch each other. Measure the diameter of the imaginary circle that includes all 3 commissures, and use a Dacron polyester fabric graft of this size to repair the root. In one end of the graft, make 3 equidistant marks (approximately $120^{\circ}$ from each other), unless the intercommissural distances are grossly different (the left cusp is usually the smallest of the 3 , and so is its intercommissural distance). By means of 3 horizontal mattress sutures (4-0 polypropylene with a fine needle), secure the Dacron polyester fabric graft inside the aortic root immediately above each commissure, with the marks made in the end of the graft as reference points. Buttress these sutures on a strip of polytetrafluoroethylene felt on the adventitia of the aortic root. As with the distal anastomosis, carefully suture the Dacron polyester fabric graft to the aortic root according to the same principles (the bites should be 3 to $4 \mathrm{~mm}$ apart and 5 to $7 \mathrm{~mm}$ into the aortic wall and the graft, and the needle is always passed from the inside to the outside of the aortic root). Once this anastomosis has been completed, inject blood cardioplegia under pressure inside the graft by clamping its distal part. This maneuver tests the integrity of the proximal anastomosis and aortic valve competence. In my experience, if the ventricle does not distend while cardioplegia is being given and the ventricular vent is not on suction, aortic insufficiency must be none or less than mild.

If the aortic sinuses are aneurysmal or extensively dissected but the cusps are normal, an aortic valve-sparing operation can be performed This is also a complicated operation, however, and I believe that an aortic root replacement is probably safer in this setting. Depending on the patients' age, you may use a mechanical or a tissue valve.

Finally, if the aortic sinuses are relatively normal with minimal dissection but the aortic cusps are diseased, replace the aortic valve, leave the coronary arteries where they are, and suture a graft in the supracoronary position. 
If the dissection involves the orifice of the right coronary artery but the intima of this artery is intact, there is no need to bypass it, because elimination of the false lumen alone should reestablish normal flow. If there is an intimal tear around the orifice of the right coronary artery or its intima is damaged, it is safer to tie the artery off and bypass it with a saphenous vein graft 1 or $2 \mathrm{~cm}$ from its origin. Remember that suturing a vein graft into collagenimpregnated Dacron polyester fabric can cause ostial stenosis of the vein as a result of pannus. Thus if the vein has a small caliber $(<4 \mathrm{~mm})$, it is safer to sew a patch of vein graft on the Dacron polyester fabric graft (a circular patch 1.5 $\mathrm{cm}^{2}$ is adequate) and then sew the saphenous vein graft onto the vein patch.

Trim the arch and aortic root grafts and suture them together. Remember that the total length of the ascending aorta graft should be only 4 to $5 \mathrm{~cm}$. Thus both the distal and the proximal grafts should be relatively short. Deair the heart and unclamp the distal graft. Reperfuse the heart, and discontinue bypass when the rectal temperature is around $36^{\circ} \mathrm{C}$. If conditions are stable and you have a cannula into the aortic graft, remove it before reversing the heparin and put it into the right atrium in the place of the venous cannula. Tie the sutures that you placed to secure the cannula in the graft, and run a fine polypropylene suture for hemostasis. Give protamine and make sure that hemostasis is perfect. Transfusions of platelets and other clotting factors are often needed in acute type A aortic dissection, because most of these patients have coagulopathy develop.

Do this operation as described, and your patient will have a $90 \%$ chance to go home alive and without neurologic deficit. You will be joining a group of "aortic surgeons" who do this operation with a mortality of $10 \%$ or less.

\section{References}

1. Chiappini B, Schepens M, Tan E, Dell' Amore A, Morshuis W, Dossche K, et al Early and late outcomes of acute type A aortic dissection: analysis of risk factors in 487 consecutive patients. Eur Heart J. 2005;26:180-6.

2. Trimarchi S, Nienaber CA, Rampoldi V, Myrmel T, Suzuki T, Mehta RH, et al Contemporary results of surgery in acute type A aortic dissection: the International Registry of Acute Aortic Dissection experience. J Thorac Cardiovasc Surg. 2005; 129:112-22.

3. Krüger T, Weigang E, Hoffmann I, Blettner M, Aebert H. GERAADA Investigators. Cerebral protection during surgery for acute aortic dissection type A: results of the German Registry for Acute Aortic Dissection Type A (GERAADA). Circulation. 2011;124:434-43.

4. Chikwe J, Cavallaro P, Itagaki S, Seigerman M, Diluozzo G, Adams DH. Nationa outcomes in acute aortic dissection: influence of surgeon and institutional volume on operative mortality. Ann Thorac Surg. 2013;95:1563-9.

5. David TE, Armstrong S, Ivanov J, Barnard S. Surgery for acute type A aortic dissection. Ann Thorac Surg. 1999;67:1999-2001; discussion 2014-29.

6. Lawton JS, Liu J, Kulshrestha K, Moon MR, Damiano RJ Jr, Maniar M, et al. The impact of surgical strategy on survival following repair of type A aortic dissection. J Thorac Cardiovasc Surg. 2015;150:294-301. 